Article

\title{
Simultaneous Generation of Two Pairs of Stokes and Terahertz Waves from Coupled Optical Parametric Oscillations with Quasi-Phase-Matching
}

\author{
Zhongyang Li * (D), Lian Tan, Yongjun Li, Bin Yuan, Silei Wang, Mengtao Wang, Pibin Bing ${ }^{(\mathbb{D}}$ and \\ Sheng Yuan \\ College of Electric Power, North China University of Water Resources and Electric Power, Zhengzhou 450045, \\ China; tanunited@ncwu.edu.cn (L.T.); liyongjun@163.com (Y.L.); x201710518296@stu.ncwu.edu.cn (B.Y.); \\ 201610521173@stu.ncwu.edu.cn (S.W.); 201610521175@stu.ncwu.edu.cn (M.W.); bing463233@163.com (P.B.); \\ shn.yuan@sohu.com (S.Y.) \\ * Correspondence: thzwave@163.com; Tel.: +86-158-3827-6960
}

Received: 23 July 2018; Accepted: 11 August 2018; Published: 14 August 2018

\begin{abstract}
We present a theoretical investigation on simultaneous generation of two pairs of Stokes and terahertz (THz) waves from coupled optical parametric oscillations (OPOs) with a quasi-phase-matching (QPM) scheme. The two pairs of Stokes and $\mathrm{THz}$ waves are generated by stimulated polariton scattering (SPS) from periodically-inverted GaP. By analyzing the QPM conditions of coupled OPOs we find that the two $\mathrm{THz}$ waves with any frequency below the transverse optical (TO) mode frequency of $\mathrm{GaP}$ can be simultaneously generated with a suitable pump wavelength. We calculate the photon flux densities of the two $\mathrm{THz}$ waves by solving the coupled wave equations. The calculation results indicate that the two $\mathrm{THz}$ waves can be efficiently generated with high pump intensities, particularly in lower $\mathrm{THz}$ frequency band.
\end{abstract}

Keywords: terahertz wave; stimulated polariton scattering; coupled optical parametric oscillation; $\mathrm{GaP}$

\section{Introduction}

In recent years, it has been demonstrated that coupled optical parametric oscillations (OPOs) can simultaneously generate two pairs of signal and idler waves from periodically-inverted $\mathrm{KTiOPO}_{4}$ (KTP) plates by a single pump wavelength [1,2]. The wavelengths for each pair of signal and idler waves satisfy quasi-phase-matching (QPM) conditions. The wavelengths of the signal and idler waves are near infrared when pump wavelength is $532 \mathrm{~nm}$. Based on the research above, we consider that the frequencies of the two idler waves generated from coupled OPOs with periodically-inverted $\mathrm{GaP}$ by stimulated polariton scattering (SPS) can extend to terahertz $(\mathrm{THz})$ frequencies. The frequency separation between the two $\mathrm{THz}$ waves can be tuned by selecting the thickness of each GaP plate. The generated two $\mathrm{THz}$ waves are useful for imaging and food inspection [3,4].

SPS which was used in $\mathrm{MgO}: \mathrm{LiNbO}_{3}, \mathrm{LiTaO}_{3}$, and $\mathrm{KTiOPO}_{4}$, has proved to be an efficient scheme to generate $\mathrm{THz}$ waves [5-12]. SPS can also be used in zinc-blende structure crystals, since the crystals have a high nonlinear coefficient and a low $\mathrm{THz}$ wave absorption coefficient $[13,14]$. GaP which has an infrared- and Raman-active transverse optical (TO) mode with frequency of $367 \mathrm{~cm}^{-1}$ is an attractive material for $\mathrm{THz}$ wave generation via SPS [15] since GaP has a high second-order nonlinear coefficient $\left(d_{36}=70.6 \mathrm{pm} / \mathrm{V}\right.$ at $\left.1064 \mathrm{~nm}\right)$ [16] and a low $\mathrm{THz}$ wave absorption coefficient below TO mode frequency [15]. Moreover, collinear configuration can be realized in GaP by using cross-Reststrahlen band dispersion compensation phase-matching [17]. 
In this work, we theoretically study simultaneous generation of two pairs of Stokes and $\mathrm{THz}$ waves from coupled OPOs with periodically-inverted GaP by SPS. The QPM conditions of the coupled OPOs are analyzed. We calculate the photon flux densities of the two $\mathrm{THz}$ waves by solving the coupled wave equations.

\section{Phase-Matching Characteristics}

In the SPS processes with cross-Reststrahlen band dispersion compensation phase-matching, pump and Stokes waves are in the near-infrared transmission window of $\mathrm{GaP}$ crystal, and $\mathrm{THz}$ wave is in the far-infrared transmission window, on the other side of the crystal's Reststrahlen band. Since the refractive indices of the three waves in $\mathrm{GaP}$ are approximately equal, the collinear phase-matching condition can be realized. Figure 1 shows $\mathrm{THz}$ frequencies $v_{\mathrm{T}}$ versus pump wavelengths $\lambda_{\mathrm{p}}$ with collinear phase-matching condition in GaP. The theoretical values of the refractive index are calculated using a Sellmeier equation for $\mathrm{GaP}$ in the infrared range [17] and in the $\mathrm{THz}$ range [15], respectively. From the figure we find that pump wavelengths $\lambda_{\mathrm{p}}$ in the range of $0.69-1.03 \mu \mathrm{m}$ fulfill collinear phase-matching, corresponding to the $\mathrm{THz}$ frequencies $v_{\mathrm{T}}$ in the range of $0.15-8.8 \mathrm{THz}$.

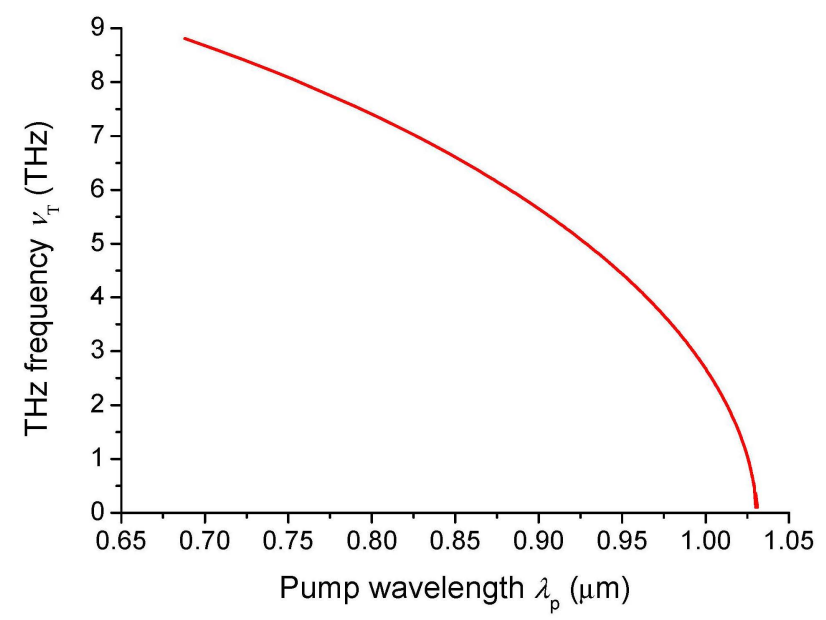

Figure 1. THz frequencies $v_{\mathrm{T}}$ versus pump wavelengths $\lambda_{\mathrm{p}}$ with collinear phase-matching condition in GaP.

Figure 2 shows phase mismatching $\Delta k$ among collinear SPS processes in GaP when $\lambda_{\mathrm{p}}=0.9 \mu \mathrm{m}$. $\Delta k=\vec{k}_{\mathrm{p}}-\vec{k}_{\mathrm{s}}-\vec{k}_{\mathrm{T}}$, where $\vec{k}_{\mathrm{p}}, \vec{k}_{\mathrm{s}}$, and $\vec{k}_{\mathrm{T}}$ are the wave vectors of pump, Stokes, and THz waves respectively. From the figure we find that $\Delta k$ is zero at $5.65 \mathrm{THz}$, where phase-matching can realize. At area I where frequencies are slightly smaller than $5.65 \mathrm{THz}, \Delta k$ is slightly larger than zero, whereas at area II where frequencies are slightly larger than $5.65 \mathrm{THz}, \Delta k$ is slightly smaller than zero. At area I the phase mismatch $\Delta k$ in optical parametric oscillation (OPO) can be compensated by grating vector $\vec{k}_{\Lambda}$ of periodically-inverted GaP. $\vec{k}_{\Lambda}=2 \pi / \Lambda$ and $\Lambda$ is the QPM period. Simultaneously, at area II, the phase mismatch $\Delta k$ in the other OPO can also be compensated by the same grating vector $\vec{k}_{\Lambda}$. As a result, the two phase mismatch of the two OPOs can be simultaneously compensated by the same grating vector $\vec{k}_{\Lambda}$. The two OPOs will couple with each other with a same pump wavelength and a same QPM period. 


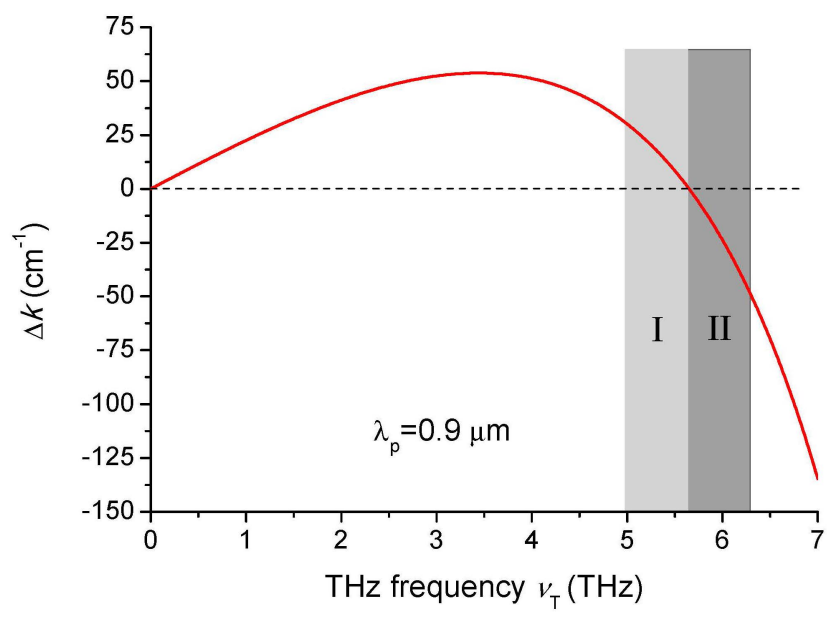

Figure 2. Phase mismatching $\Delta k$ versus $\mathrm{THz}$ frequencies $v_{\mathrm{T}}$ with collinear phase-matching condition in $\mathrm{GaP}, \lambda_{\mathrm{p}}=0.9 \mu \mathrm{m}$.

In order to achieve efficient conversion of coupled OPOs from pump wave to $\mathrm{THz}$ wave, a precise phase-matching condition must be satisfied. Figure 3 shows the QPM scheme of coupled OPOs with periodically-inverted GaP. In order to simultaneously satisfy the two QPM conditions, accurate value of grating vector $\vec{k}_{\Lambda}$ is required. The two QPM conditions of the coupled OPOs are

$$
\begin{aligned}
& \vec{k}_{\mathrm{p}}-\vec{k}_{\mathrm{s} 1}-\vec{k}_{\mathrm{T} 1}+\vec{k}_{\Lambda}=0 \\
& \vec{k}_{\mathrm{p}}-\vec{k}_{\mathrm{s} 2}-\vec{k}_{\mathrm{T} 2}-\vec{k}_{\Lambda}=0
\end{aligned}
$$

where $\vec{k}_{\mathrm{s} 1}$ and $\vec{k}_{\mathrm{s} 2}$ are the wave vectors of the two Stokes waves respectively, $\vec{k}_{\mathrm{T} 1}$ and $\vec{k}_{\mathrm{T} 2}$ are the wave vectors of the two $\mathrm{THz}$ waves respectively. The energy conservation condition has to be fulfilled,

$$
\begin{aligned}
& \frac{1}{\lambda_{\mathrm{p}}}-\frac{1}{\lambda_{\mathrm{s} 1}}-\frac{1}{\lambda_{\mathrm{T} 1}}=0 \\
& \frac{1}{\lambda_{\mathrm{p}}}-\frac{1}{\lambda_{\mathrm{s} 2}}-\frac{1}{\lambda_{\mathrm{T} 2}}=0
\end{aligned}
$$

where $\lambda_{\mathrm{s} 1}$ and $\lambda_{\mathrm{s} 2}$ are the wavelengths of the two Stokes waves respectively, $\lambda_{\mathrm{T} 1}$ and $\lambda_{\mathrm{T} 2}$ are the wavelengths of the two $\mathrm{THz}$ waves respectively. If the two QPM conditions of the coupled OPOs are simultaneously satisfied, the two $\mathrm{THz}$ waves can be simultaneously generated with a single pump wave. Figure 4 shows $\mathrm{THz}$ frequencies and Stokes wavelengths versus QPM period $\Lambda$ with coupled OPOs with different pump wavelengths. $v_{\mathrm{T} 1}$ and $v_{\mathrm{T} 2}$ are frequencies of the two THz waves, $v_{\mathrm{T} 1}=c / \lambda_{\mathrm{T} 1}$ and $v_{\mathrm{T} 2}=c / \lambda_{\mathrm{T} 2}, c$ is velocity of light. From the figure we find that two pairs of Stokes and $\mathrm{THz}$ waves can be generated by coupled OPOs with a single pump wave. As QPM period $\Lambda$ changes the $\mathrm{THz}$ frequencies and Stokes wavelengths are tuned. The difference frequencies between the two $\mathrm{THz}$ waves become small as QPM period $\Lambda$ increases. The minimum difference frequencies are smaller than $0.01 \mathrm{THz}$. As pump wavelengths change from $0.75 \mu \mathrm{m}$ to $0.852,0.95$, and $1.025 \mu \mathrm{m}$, $v_{\mathrm{T} 1}$ and $v_{\mathrm{T} 2}$ decrease from $8.1 \mathrm{THz}$ to $1.05 \mathrm{THz}$. From Figure 4 we conclude that the two $\mathrm{THz}$ waves with any frequency from $1.05 \mathrm{THz}$ to $8.1 \mathrm{THz}$ can be simultaneously generated by coupled OPOs with a suitable pump wavelength. 


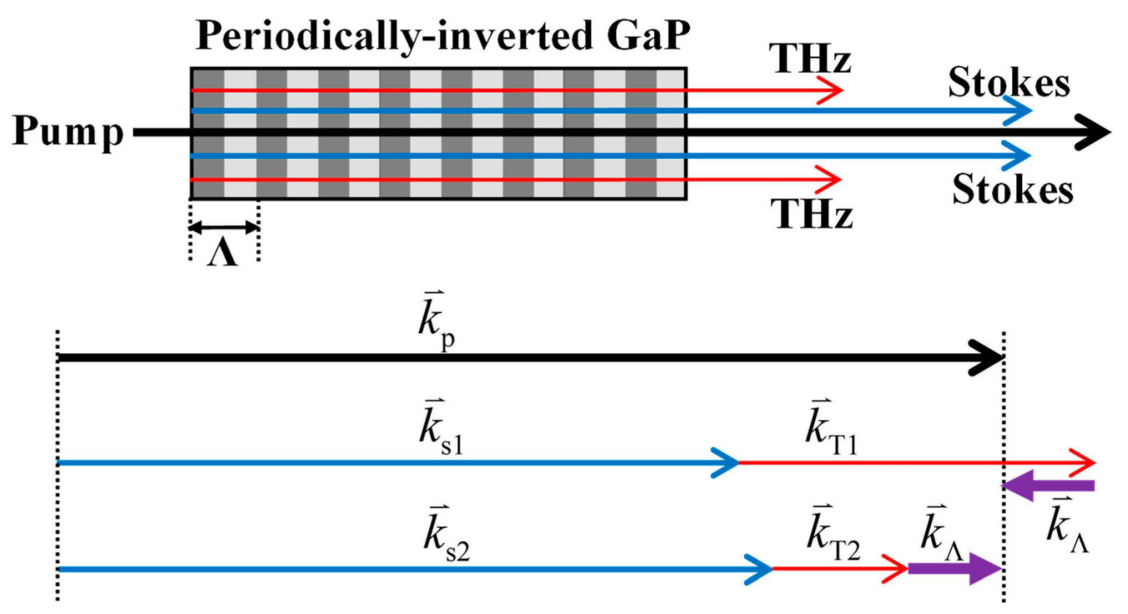

Figure 3. Schematic diagram of QPM scheme of the coupled OPOs with periodically-inverted GaP. Pump wave, Stokes waves, and THz waves are collinear. $\vec{k}_{\mathrm{p}}$ is the wave vector of pump wave and $\vec{k}_{\mathrm{s} 1}$ and $\vec{k}_{\mathrm{s} 2}$ are the wave vectors of the two Stokes waves respectively, $\vec{k}_{\mathrm{T} 1}$ and $\vec{k}_{\mathrm{T} 2}$ are the wave vectors of the two THz waves respectively, $\vec{k}_{\Lambda}$ is the grating vector of periodically-inverted GaP.
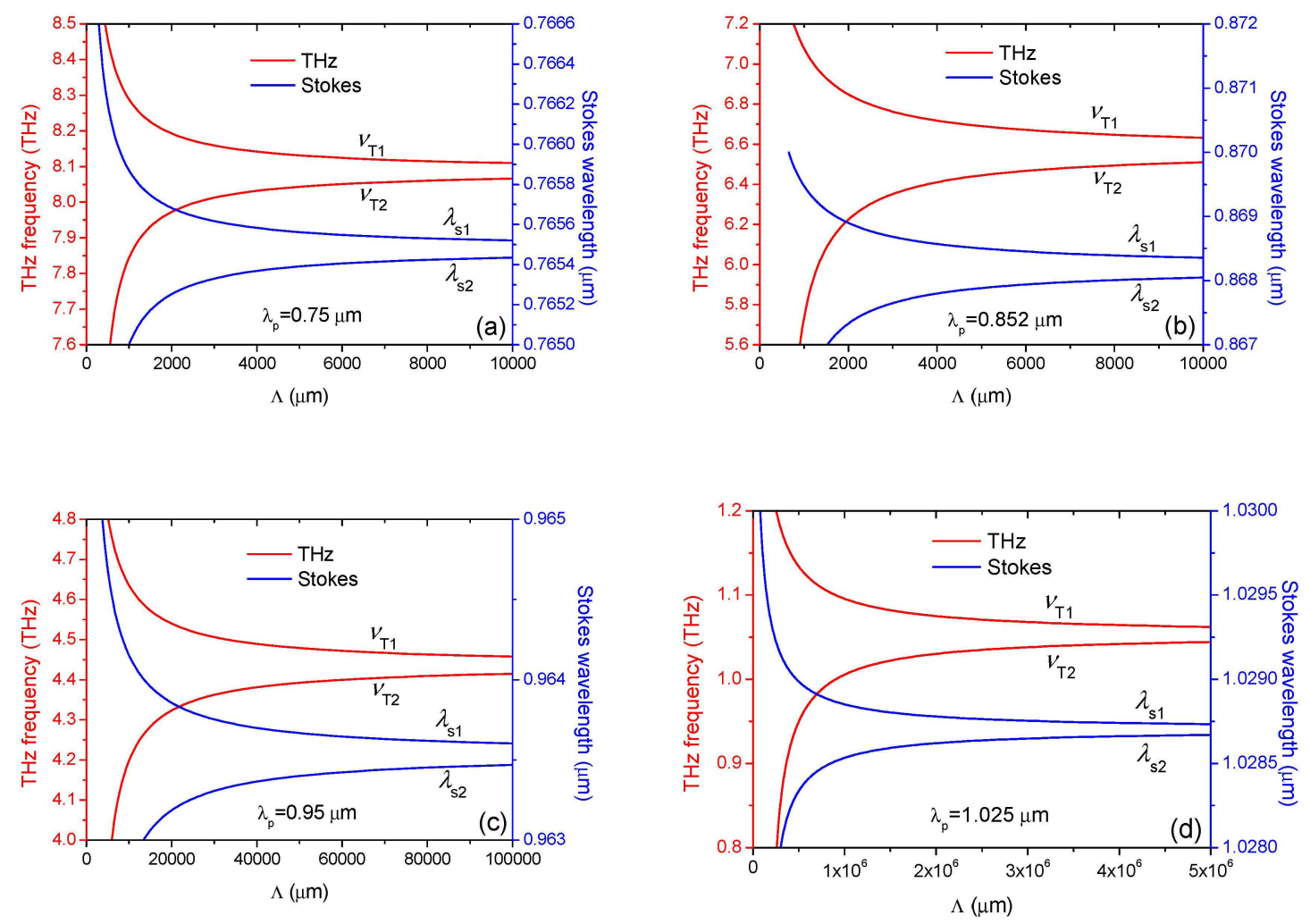

Figure 4. THz frequencies and Stokes wavelengths versus QPM period $\Lambda$ with coupled OPOs. (a) $\lambda_{\mathrm{p}}=0.75 \mu \mathrm{m}$; (b) $\lambda_{\mathrm{p}}=0.852 \mu \mathrm{m} ;$ (c) $\lambda_{\mathrm{p}}=0.95 \mu \mathrm{m}$; (d) $\lambda_{\mathrm{p}}=1.025 \mu \mathrm{m}$.

\section{THz Wave Photon Flux Density}

The generated THz intensities by SPS processes depend on the nonlinear optical susceptibility and $\mathrm{THz}$ absorption coefficients. The nonlinear optical susceptibility in $\mathrm{GaP}$ in the $\mathrm{THz}$ frequency range is governed by the superposition of electronic and ionic contributions. Faust showed that the ionic and electronic contributions are of opposite sign, leading to a cancellation of both contributions below the TO mode frequency of GaP [18]. Sussman deduced coupled wave equations for SPS processes [19]. 
In this work we calculate $\mathrm{THz}$ intensity based on the theories of Faust and Sussman. The analytical expression of $\mathrm{THz}$ parametric gain coefficient $g_{\mathrm{T}}$ in SPS processes under the QPM condition in the international system of units can be written as $[19,20]$

$$
\begin{gathered}
g_{\mathrm{T}}=\frac{\alpha_{\mathrm{T}}}{2}\left\{\left[1+16\left(\frac{g_{0}}{\alpha_{\mathrm{T}}}\right)^{2}\right]^{\frac{1}{2}}-1\right\} \\
g_{0}^{2}=\frac{\omega_{\mathrm{s}} \omega_{\mathrm{T}}}{128 \pi^{2} \varepsilon_{0} c^{3} n_{\mathrm{p}} n_{\mathrm{s}} n_{\mathrm{T}}} I_{\mathrm{p}} d_{e f f}^{2} \\
d_{e f f}=d_{e}\left(1+C_{1} \frac{\omega_{\mathrm{TO}}^{2}}{\omega_{\mathrm{TO}}^{2}-\omega_{\mathrm{T}}^{2}-i \omega_{\mathrm{T}} \Gamma}\right) \\
\alpha_{\mathrm{T}}=2 \frac{\omega_{\mathrm{T}}}{c} \operatorname{Im}\left(\varepsilon_{\infty}+\frac{S \omega_{\mathrm{TO}}^{2}}{\omega_{\mathrm{TO}}^{2}-\omega_{\mathrm{T}}^{2}-i \omega_{\mathrm{T}} \Gamma}\right)^{\frac{1}{2}}
\end{gathered}
$$

where $\omega_{\mathrm{TO}}, S$, and $\Gamma$ denote eigenfrequency, oscillator strength of the polariton modes, and the bandwidth of the TO mode in GaP crystal, respectively. $\omega_{\mathrm{S}}$ and $\omega_{\mathrm{T}}$ are the angular frequencies of Stoke and $\mathrm{THz}$ waves, respectively. $\varepsilon_{0}$ and $\varepsilon_{\infty}$ are vacuum dielectric constant and high-frequency dielectric constant, respectively. $I_{\mathrm{p}}$ is the power density of pump wave, $g_{0}$ is the low-loss parametric gain. $n_{\mathrm{p}}$, $n_{\mathrm{S}}$, and $n_{\mathrm{T}}$ are the refractive indices of the pump, Stokes and THz waves, respectively. $\alpha_{\mathrm{T}}$ is material absorption coefficient in THz region. $d_{e}$ is the electronic second-order nonlinear coefficient, and $d_{e f f}$ is the bulk value of the second-order nonlinear coefficient involving electronic and ionic contributions. $C_{1}$ is a coupling constant, and $C_{1}$ is -0.53 for GaP [18]. Figure 5 shows calculated $d_{e f f}$ and $\alpha_{\mathrm{T}}$ versus $\mathrm{THz}$ frequencies. In the calculation $d_{e}$ is $70.6 \mathrm{pm} / \mathrm{V}$ with pump wavelength of $1.064 \mu \mathrm{m}$. From the figure we find that $\alpha_{\mathrm{T}}$ monotonously increases with frequencies, and $d_{e f f}$ smoothly decreases first and then rapidly increases. $d_{e f f}$ is smaller than $70.6 \mathrm{pm} / \mathrm{V}$ below the TO mode frequency of GaP due to the cancellation of ionic and electronic contributions. The minimum value of $d_{e f f}$ is $5.0 \mathrm{pm} / \mathrm{V}$ at $7.54 \mathrm{THz}$. When approaching TO mode frequency, $d_{e f f}$ is larger than $70.6 \mathrm{pm} / \mathrm{V}$ because polaritons at this area induce giant ionic nonlinearities. By comprehensive consideration of $d_{e f f}$ and $\alpha_{\mathrm{T}}$ we conclude that the lower frequencies of $\mathrm{THz}$ wave in the range of $0-7.54 \mathrm{THz}$, the higher intensities of $\mathrm{THz}$ wave generated from SPS processes.

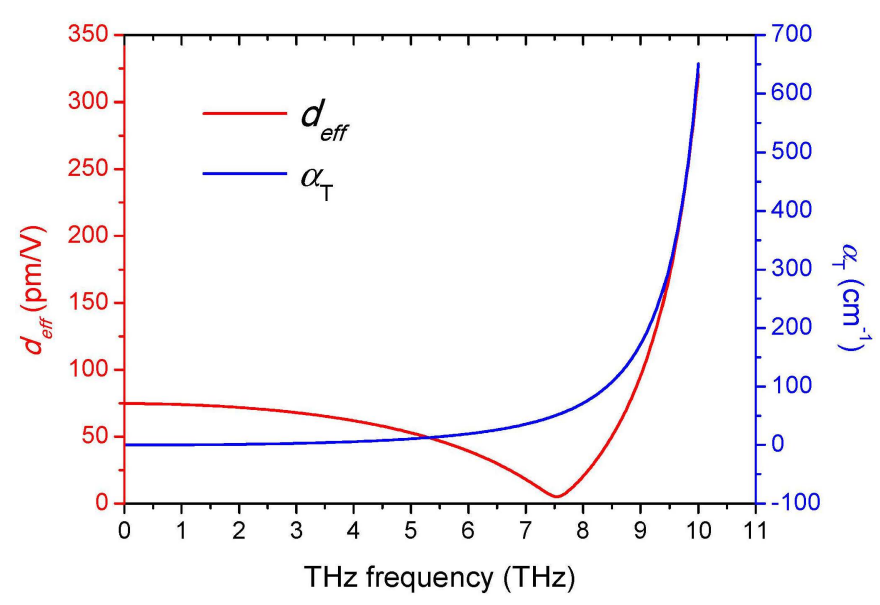

Figure 5. Calculated $d_{e f f}$ and $\alpha_{\mathrm{T}}$ versus THz frequencies. $d_{e}$ is $70.6 \mathrm{pm} / \mathrm{V}$ with pump wavelength of $1.064 \mu \mathrm{m}$. 
With $\mathrm{THz}$ wave absorption, without phase mismatch and pump depletion, the coupled wave equations can be solved to give the THz photon flux density $\phi_{\mathrm{T}}$ with a general solution $[21,22]$, given by

$$
\phi_{\mathrm{T}}=\phi_{\mathrm{s}}(0) e^{-\alpha_{\mathrm{T}} L / 2} \frac{g_{\mathrm{T}}^{2}}{g_{\mathrm{T}}^{2}+\left(\frac{\alpha_{\mathrm{T}}}{4}\right)^{2}} \times\left|\sinh \left(\sqrt{g_{\mathrm{T}}^{2}+\left(\frac{\alpha_{\mathrm{T}}}{4}\right)^{2}} L\right)\right|^{2}
$$

where $L$ is the crystal length. The initial THz photon flux density $\phi_{\mathrm{T}}$ is assumed to be zero, and $\phi_{\mathrm{s}}(0)$ is the initial Stokes wave photon flux density. Figure 6 shows THz wave photon flux density $\phi_{\mathrm{T}} / \phi_{\mathrm{s}}(0)$ from coupled OPOs by SPS processes. $\phi_{\mathrm{T} 1}$ and $\phi_{\mathrm{T} 2}$ are the photon flux densities of $v_{\mathrm{T} 1}$ and $v_{\mathrm{T} 2}$, respectively, $\phi_{\mathrm{s} 1}(0)$ and $\phi_{\mathrm{s} 2}(0)$ are the initial photon flux densities of $\lambda_{\mathrm{s} 1}$ and $\lambda_{\mathrm{s} 2}$, respectively. In the calculations pump intensity $I_{\mathrm{p}}$ is $500 \mathrm{MW} / \mathrm{cm}^{2}$ since the damage threshold of $\mathrm{GaP}$ is $650 \mathrm{MW} / \mathrm{cm}^{2}$ [23]. From Figure 6a we find that $\phi_{\mathrm{T}} / \phi_{\mathrm{s}}(0)$ rapidly increases with crystal length $L$ as $v_{\mathrm{T} 1}=1.13 \mathrm{THz}$ and $v_{\mathrm{T} 2}=0.95 \mathrm{THz}$. As shown in Figure $5, d_{\text {eff }}$ is large and $\alpha_{\mathrm{T}}$ is small as $\mathrm{THz}$ wave frequencies are around $1 \mathrm{THz}$. The large $d_{\text {eff }}$ and small $\alpha_{\mathrm{T}}$ enhance the rapid increase of $\phi_{\mathrm{T}} / \phi_{\mathrm{s}}(0)$ with crystal length $L$. As the coefficient $\alpha_{\mathrm{T}}$ of $1.13 \mathrm{THz}$ is larger than that of $0.95 \mathrm{THz}, \phi_{\mathrm{T} 1} / \phi_{\mathrm{s} 1}(0)$ is smaller than $\phi_{\mathrm{T} 2} / \phi_{\mathrm{s} 2}(0)$. $\phi_{\mathrm{T} 2} / \phi_{\mathrm{s} 2}(0)$ with value of 0.016 can be reached as crystal length $L$ is $5 \mathrm{~cm}$. From Figure $6 \mathrm{~b}$ we find that $\phi_{\mathrm{T}} / \phi_{\mathrm{S}}(0)$ rapidly increases with crystal length $L$ and then keeps invariant as $v_{\mathrm{T} 1}=6.69 \mathrm{THz}$ and $v_{\mathrm{T} 2}=6.45 \mathrm{THz}$. Compared with Figure $6 \mathrm{a}, d_{\text {eff }}$ becomes smaller and $\alpha_{\mathrm{T}}$ becomes larger, which induces an intensive decrease of $\phi_{\mathrm{T} 1} / \phi_{\mathrm{s} 1}(0)$ and $\phi_{\mathrm{T} 2} / \phi_{\mathrm{s} 2}(0)$ in Figure $6 \mathrm{~b}$. From Figure 6 we conclude that high-power $\mathrm{THz}$ waves can be generated in lower $\mathrm{THz}$ frequency band. The intensity of $\mathrm{THz}$ wave can be boosted by injecting an initial Stokes wave as a seed light.
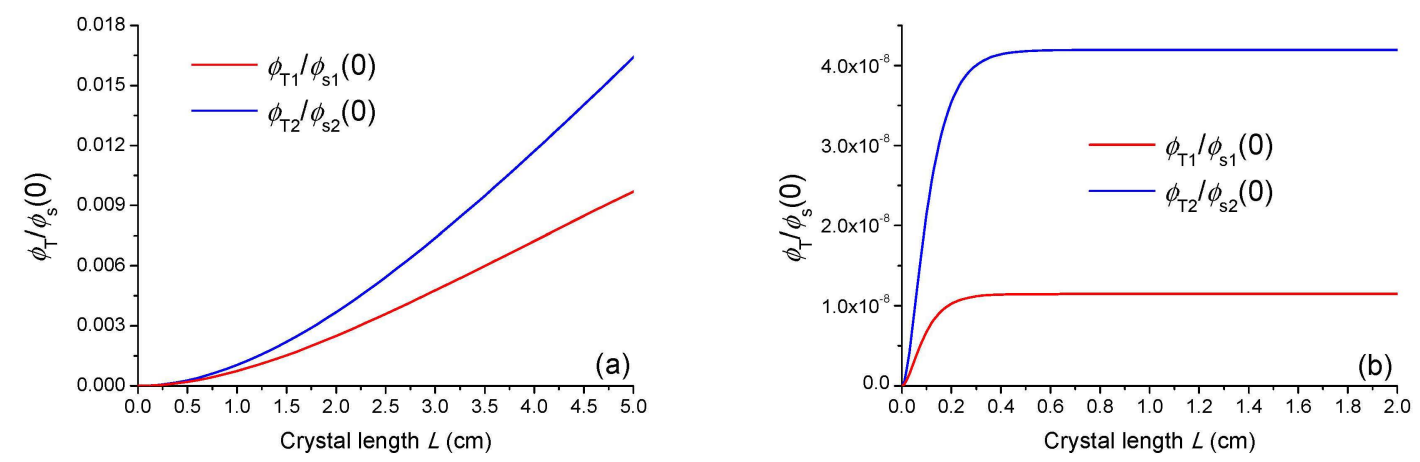

Figure 6. THz wave photon flux density $\phi_{\mathrm{T}} / \phi_{\mathrm{s}}(0)$ versus crystal length, $I_{\mathrm{p}}=500 \mathrm{MW} / \mathrm{cm}^{2}$. (a) $\lambda_{\mathrm{p}}=1.025 \mu \mathrm{m}, v_{\mathrm{T} 1}=1.13 \mathrm{THz}, v_{\mathrm{T} 2}=0.95 \mathrm{THz}, \Lambda=500,000 \mu \mathrm{m}$; (b) $\lambda_{\mathrm{p}}=0.852 \mu \mathrm{m}, \nu_{\mathrm{T} 1}=6.69 \mathrm{THz}$, $v_{\mathrm{T} 2}=6.45 \mathrm{THz}, \Lambda=5000 \mu \mathrm{m}$.

As shown in Equation (6), low-loss parametric gain $g_{0}$ is proportional to the pump intensity $I_{\mathrm{p}}$. A pump wave with a pump intensity on the order of $\mathrm{GW} / \mathrm{cm}^{2}$ can enlarge the gain coefficient $g_{\mathrm{T}}$ by several orders of magnitude [24,25], which can enhance the intensity of $\mathrm{THz}$ wave. Figure 7 shows the THz wave photon flux density $\phi_{\mathrm{T}} / \phi_{\mathrm{s}}(0)$ versus pump intensity $I_{\mathrm{p}} \cdot \phi_{\mathrm{T} 1} / \phi_{\mathrm{s} 1}(0)$ and $\phi_{\mathrm{T} 2} / \phi_{\mathrm{s} 2}(0)$ monotonously increase with the increase of pump intensity $I_{\mathrm{p}} . \phi_{\mathrm{T} 2} / \phi_{\mathrm{s} 2}(0)$, with a value of 0.026 , can be reached as pump intensity $I_{\mathrm{p}}$ is $650 \mathrm{MW} / \mathrm{cm}^{2}$. The intense $\mathrm{THz}$ wave can be generated using a pump wave with a high intensity. 


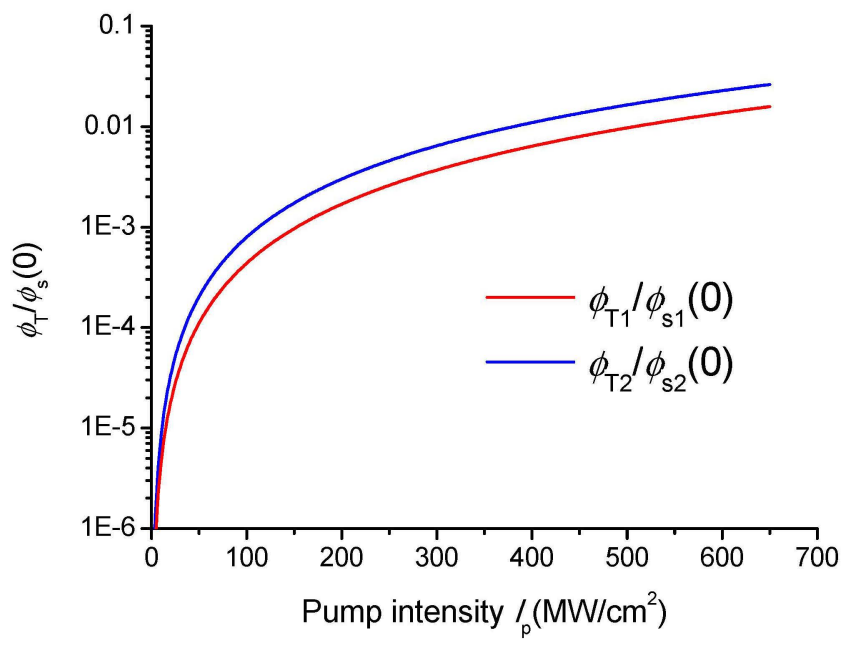

Figure 7. THz wave photon flux density $\phi_{\mathrm{T}} / \phi_{\mathrm{s}}(0)$ versus pump intensity $I_{\mathrm{p}}, L=5 \mathrm{~cm}, \lambda_{\mathrm{p}}=1.025 \mu \mathrm{m}$, $v_{\mathrm{T} 1}=1.13 \mathrm{THz}, v_{\mathrm{T} 2}=0.95 \mathrm{THz}, \Lambda=500,000 \mu \mathrm{m}$.

Compared with other SPS processes generating THz waves, the scheme in this work generating two pairs of Stokes and THz waves from coupled OPOs with periodically-inverted GaP by SPS processes has four advantages. First, two pairs of Stokes and $\mathrm{THz}$ waves are simultaneously generated only by a periodically-inverted GaP crystal and a pump laser. Second, since the two pairs of Stokes and $\mathrm{THz}$ waves are simultaneously generated by a same pump wave, the two pairs of Stokes and $\mathrm{THz}$ waves are phase-conjugate. Third, the two $\mathrm{THz}$ waves with any frequency below the TO mode frequency of $\mathrm{GaP}$ can be simultaneously generated with a suitable pump wavelength. Fourth, the intensities of the THz waves can be enhanced by injecting an initial Stokes wave as a seed light. The scheme can be applied to other crystals, such as GaAs, ZnTe, CdTe, DSTMS (4-N,N-dimethylamino-4'- $N^{\prime}$-methylstilbazolium 2,4,6-trime-thylbenzenesulfonate), and $\mathrm{OH} 1$ (2-[3-(4-hydroxystyryl)-5,5-dimethylcyclohex-2-enylidene]malononitrile). These crystals can generate $\mathrm{THz}$ wave by SPS with collinear phase-matching configuration. Moreover, these crystals can be periodically-inverted or periodically poled to satisfy QPM condition. Furthermore, THz absorption coefficients of these crystals are small.

\section{Conclusions}

Two pairs of Stokes and THz waves can be simultaneously generated from coupled OPOs with SPS processes in periodically-inverted GaP. The two QPM conditions of the coupled OPOs are simultaneously satisfied. The two THz waves with any frequency below the TO mode frequency of $\mathrm{GaP}$ can be generated with a suitable pump wavelength. THz waves can be efficiently generated with high pump intensities, particularly in a lower $\mathrm{THz}$ frequency band.

Author Contributions: Conceptualization, Z.L., L.T., and Y.L. Formal analysis, B.Y.; Investigation, P.B.; Software, S.W. and S.Y.; Visualization, M.W., Z.L. wrote the manuscript. All authors read and approved the final version of the manuscript.

Funding: This work was supported by the National Natural Science Foundation of China (61601183); the Natural Science Foundation of Henan Province (162300410190); the Program for Innovative Talents (in Science and Technology) in University of Henan Province (18HASTIT023); the Young Backbone Teachers in University of Henan Province (2014GGJS-065); and the Program for Innovative Research Team (in Science and Technology) in University of Henan Province (16IRTSTHN017).

Conflicts of Interest: The authors declare no conflicts of interest. The founding sponsors had no role in the design of the study; in the collection, analyses, or interpretation of the data; in the writing of the manuscript; or in the decision to publish the results. 


\section{References}

1. Mu, X.D.; Meissner, H.; Lee, H.C. Optical parametric oscillations of $2 \mu \mathrm{m}$ in multiple-layer bonded walk-off compensated KTP stacks. Opt. Lett. 2010, 35, 387-389. [CrossRef] [PubMed]

2. Zhao, P.; Ragam, S.; Ding, Y.J.; Zotova, I.B.; Mu, X.D.; Lee, H.C.; Meissner, S.K.; Meissner, H. Singly resonant optical parametric oscillator based on adhesive-free-bonded periodically inverted $\mathrm{KTiOPO}_{4}$ plates: Terahertz generation by mixing a pair of idler waves. Opt. Lett. 2012, 37, 1283-1285. [CrossRef] [PubMed]

3. Valk, N.C.J.; Marel, W.A.M.; Planken, P.C.M. Terahertz polarization imaging. Opt. Lett. 2005, 30, $2802-2804$. [CrossRef] [PubMed]

4. Mathanker, S.K.; Weckler, P.R.; Wang, N. Terahertz (THz) applications in food and agriculture: A review. Trans. ASABE 2013, 56, 1213-1226.

5. Kawase, K.; Shikata, J.; Ito, H. Terahertz wave parametric source. J. Phys. D Appl. Phys. 2002, 35, R1-R14. [CrossRef]

6. Ikari, T.; Guo, R.; Minamide, H.; Ito, H. Energy scalable terahertz-wave parametric oscillator using surface-emitted configuration. J. Eur. Opt. Soc. Rapid Publ. 2010, 5, 10054.

7. Ortega, T.A.; Pask, H.M.; Spence, D.J.; Lee, A.J. THz polariton laser using an intracavity $\mathrm{Mg}: \mathrm{LiNbO}_{3}$ crystal with protective Teflon coating. Opt. Express 2017, 25, 3991-3999. [CrossRef] [PubMed]

8. Zhang, R.L.; Qu, Y.C.; Zhao, W.J.; Chen, Z.L. High energy, widely tunable Si-prism-array coupled terahertz-wave parametric oscillator with a deformed pump and optimal crystal location for angle tuning. Appl. Opt. 2017, 56, 2412-2417. [CrossRef] [PubMed]

9. Molter, D.; Theuer, M.; Beigang, R. Nanosecond terahertz optical parametric oscillator with a novel quasiphase-matching scheme in lithium niobate. Opt. Express 2009, 17, 6623-6628. [CrossRef] [PubMed]

10. Jang, H.; Strömqvist, G.; Pasiskevicius, V.; Canalias, C. Control of forward stimulated polariton scattering in periodically-poled KTP crystals. Opt. Express 2013, 21, 27277-27283. [CrossRef] [PubMed]

11. Jang, H.; Viotti, A.L.; Strömqvist, G.; Zukauskas, A.; Canalias, C.; Pasiskevicius, V. Counter-propagating parametric interaction with phonon-polaritons in periodically poled $\mathrm{KTiOPO}_{4}$. Opt. Express 2017, 25, 2677-2686. [CrossRef] [PubMed]

12. Wu, M.H.; Chiu, Y.C.; Wang, T.D.; Zhao, G.; Zukauskas, A.; Laurell, F.; Huang, Y.C. Terahertz parametric generation and amplification from potassium titanyl phosphate in comparison with lithium niobate and lithium tantalate. Opt. Express 2016, 24, 25964-25973. [CrossRef] [PubMed]

13. Vodopyanov, K.L. Optical THz-wave generation with periodically-inverted GaAs. Laser Photon. Rev. 2008, 2, 11-25. [CrossRef]

14. Vodopyanov, K.L. Terahertz-wave generation with periodically inverted gallium arsenide. Laser Phys. 2009, 19, 305-321. [CrossRef]

15. Palik, E.D. Handbook of Optical Constants of Solids III; Elsevier: San Diego, CA, USA, 1998; pp. 32-40.

16. Shoji, I.; Kondo, T.; Ito, R. Second-order nonlinear susceptibilities of various dielectric and semiconductor materials. Opt. Quantum Electron. 2002, 34, 797-833. [CrossRef]

17. Bahoura, M.; Herman, G.S.; Barnes, N.P.; Bonner, C.E.; Higgins, P.T. Terahertz wave source via difference-frequency mixing using cross-Reststrahlen band dispersion compensation phase matching: A material study. Proc. SPIE 2000, 3928, 132-140.

18. Faust, W.L.; Henry, C.H. Mixing of visible and near-resonance infrared light in GaP. Phys. Rev. Lett. 1966, 17, 1265-1268. [CrossRef]

19. Sussman, S.S. Tunable Light Scattering from Transverse Optical Modes in Lithium Niobate; Report No. su-mlr-1851(1970); Microwave Laboratory, Stanford University: Stanford, CA, USA, 1971.

20. Walsh, D.A. Intracavity Terahertz Optical Parametric Oscillators. Ph.D. Thesis, University of St Andrews, Scotland, UK, 2011.

21. Kitaeva, G.K.; Penin, A.N. Parametric frequency conversion in layered nonlinear media. J. Exp. Theor. Phys. 2004, 98, 272-286. [CrossRef]

22. Wang, T.D.; Huang, Y.C.; Chuang, M.Y.; Lin, Y.H.; Lee, C.H.; Lin, Y.Y.; Lin, F.Y.; Kitaeva, G.K. Long range parametric amplification of $\mathrm{THz}$ wave with absorption loss exceeding parametric gain. Opt. Express 2013, 21, 2452-2462. [CrossRef] [PubMed]

23. Gonzalez, L.P.; Guha, S.; Trivedi, S. Damage thresholds and nonlinear optical performance of GaP. In Proceedings of the Conference on Lasers and Electro-Optics, San Francisco, CA, USA, 16-21 May 2004. 
24. Takida, Y.; Shikata, J.; Nawata, K.; Tokizane, Y.; Han, Z.L.; Koyama, M.; Notake, T.; Hayashi, S.; Minamide, H. Terahertz-wave parametric gain of stimulated polariton scattering. Phys. Rev. A 2016, 93, 043836. [CrossRef]

25. Hayashi, S.; Nawata, K.; Taira, T.; Shikata, J.; Kawase, K.; Minamide, H. Ultrabright continuously tunable terahertz-wave generation at room temperature. Sci. Rep. 2014, 4, 5045. [CrossRef] [PubMed] 